\title{
Correction to: High-Dimensional Immunophenotyping with 37-Color Panel Using Full-Spectrum Cytometry
}

\author{
Marco A. Fernandez, Hammad Alzayat, Maria C. Jaimes, \\ Yacine Kharraz, Gerard Requena, and Pedro Mendez
}

Correction to:

Chapter 4 in: Aik T. Ooi (ed.), Single-Cell Protein Analysis:

Methods and Protocols, Methods in Molecular Biology, vol. 2386, https://doi.org/10.1007/978-1-0716-1771-7_4

The chapter was inadvertently published with incorrect figure legends, reference citations, and order of references.

These errors have been corrected by updating the correct figure legends, reference citations, and the order of references as seen below:

Figure 2 Legend was: Example of population selection for unmixing on single stain and unstained reference controls. Spectral plot (right) shows specific signature of each fluorochrome and cellular autofluorescence

Figure 2 Legend has been corrected as: Anti-IgG antibody cross-reacts with anti-TCR $\mathrm{Vd} 2$ (A) and anti-IgA (B). Left plots, protocol with simultaneous staining; right plots, modified protocol with sequential staining

Figure 3 Legend was: Manual Gating Strategy, part 1

Figure 3 Legend has been corrected as: CD19 SparNIR685 signal pre- and postfixation step. Post-fixation staining shows better resolution of CD19+ cells

Figure 4 Legend was: Manual Gating Strategy, part 2

Figure 4 Legend has been corrected as: Example of population selection for unmixing on single stain and unstained reference controls. Spectral plot (right) shows specific signature of each fluorochrome and cellular autofluorescence

Figure 5 Legend was: Manual Gating Strategy, part 3

Figure 5 Legend has been corrected as: Manual Gating Strategy, part 1

The updated online version of the chapter can be found at https://doi.org/10.1007/978-1-0716-1771-7_4

Aik T. Ooi (ed.), Single-Cell Protein Analysis: Methods and Protocols, Methods in Molecular Biology, vol. 2386 https://doi.org/10.1007/978-1-0716-1771-7_18, ( ) The Author(s), under exclusive license to Springer Science+Business Media, LLC, part of Springer Nature 2022, Corrected Publication 2022 
Figure 6 Legend was: Visualization of high-dimensional data using t-SNE and UMAP algorithms on PBMC -excluding monocytes- from three concatenated samples. A. Biaxial plot with t-SNE (upper) and UMAP (lower) maps. B. Overlayed color-coded expression of several markers (CD3, CD4, CD8, TIGIT, CD57, CD19, IgM, IgD, CXCR5, CCR6, CD56, CD16 CD161, CD123 and CD27) on dimensional reduction t-SNE map. Data generated with the OMIQ Platform (

Figure 6 Legend has been corrected as: Manual Gating Strategy, part 2

Figure 7 Legend was: Anti-IgG antibody cross-reacts with anti-TCR Vd2 (A) and antiIgA (B). Left plots, standard protocol with simultaneous staining; right plots, alternative staining with sequential staining

Figure 7 Legend has been corrected as: Manual Gating Strategy, part 3

Figure 8 Legend was: CD19 SparNIR685 signal pre- and post-fixation step. Postfixation staining shows better resolution of CD19+ cells

Figure 8 Legend has been corrected as: Visualization of high-dimensional data using $\mathrm{t}$-SNE and UMAP algorithms on PBMC - excluding monocytes - from three concatenated samples. (A) Biaxial plot with t-SNE (upper) and UMAP (lower) maps. (B) Overlayed colorcoded expression of several markers (CD3, CD4, CD8, TIGIT, CD57, CD19, IgM, IgD, CXCR5, CCR6, CD56, CD16, CD161, CD123, and CD27) on dimensional reduction t-SNE map. Data generated with the OMIQ Platform (https://omiq.ai)

The following citations have been revised:

\section{Section 3 : Methods}

[8] has been updated as (see Notes 9 and 10)

[9] has been updated as [8]

[10] has been updated as [9]

$[8,11]$ has been updated as $[10,11]$

\section{Section 4 Notes}

3. [14] has been updated as [13]

5. [15] has been updated as [14]

7. $[9,16]$ has been update as $[\mathbf{8}, \mathbf{1 5}]$

9. [17] has been updated as [16]

10. [17] has been updated as [16]

11. $[15,18,19]$ has been updated as [17-19]

References have been sorted in the following order in the updated version of the chapter.

1. Herzenberg LA, Sweet RG, Herzenberg LA (1976) Fluorescence-activated cell sorting. Sci Am 234(3):108-117. https://doi.org/10.1038/scientificamerican0376-108

2. Biolegend History of Flow Cytometry. https://www.biolegend.com/en-us/history-offlow

3. Herzenberg LA, Parks D, Sahaf B, et al (2002) The history and future of the fluorescence activated cell sorter and flow cytometry: a view from Stanford. Clin Chem 48: 1819-1827. https://doi.org/10.1093/clinchem/48.10.1819

4. Hu B, Guo H, Zhou P, Shi ZL (2020) Characteristics of SARS-CoV-2 and COVID-19. Nat Rev Microbiol 19(3):141-154. https://doi.org/10.1038/s41579-020-00459-7 
5. Nicola M, Alsafi Z, Sohrabi C, et al (2020) The socio-economic implications of the coronavirus pandemic (COVID-19): a review. Int J Surg 78:185-193

6. John Hopkins Coronavirus Resource Center

7. Nolan JP, Condello D (2013) Spectral flow cytometry. Curr Protoc Cytom. https:// doi.org/10.1002/0471142956.cy0127s63

8. Mahnke YD, Roederer M (2007) Optimizing a multicolor immunophenotyping assay. Clin Lab Med 27(3):469-485, v

9. Hulspas R (2010) Titration of fluorochrome-conjugated antibodies for labeling cell surface markers on live cells. Curr Protoc Cytom. https://doi.org/10.1002/ 0471142956.cy0629s54

10. Niewold P, Ashhurst TM, Smith AL, King NJC (2020) Evaluating spectral cytometry for immune profiling in viral disease. Cytom Part A 97(11):1165-1179. https://doi. org/10.1002/cyto.a.24211

11. Schmutz S, Valente M, Cumano A, Novault S (2016) Spectral cytometry has unique properties allowing multicolor analysis of cell suspensions isolated from solid tissues. PLoS One 11(8):e0159961. https://doi.org/10.1371/journal.pone.0159961

12. Interim Laboratory Biosafety Guidelines for Handling and Processing Specimens Associated with Coronavirus Disease 2019 (COVID-19). Centers Dis Control Prevention

13. Zhao Q, Meng M, Kumar R, et al (2020) Lymphopenia is associated with severe coronavirus disease 2019 (COVID-19) infections: a systemic review and meta-analysis. Int J Infect Dis 96:131-135. https://doi.org/10.1016/j.ijid.2020.04.086

14. Jalbert E, Shikuma CM, Ndhlovu LC, Barbour JD (2013) Sequential staining improves detection of CCR2 and CX3CRl on monocytes when simultaneously evaluating CCR5 by multicolor flow cytometry. Cytometry A 83(3):280-286.. https://doi.org/10. $1002 /$ cyto.a.22257

15. Ferrer-Font L, Pellefigues C, Mayer JU, et al (2020) Panel design and optimization for high-dimensional immunophenotyping assays using spectral flow cytometry. Curr Protoc Cytom 92:1-25. https://doi.org/10.1002/cpcy.70

16. Park LM, Lannigan J, Jaimes MC (2020) Forty-color full spectrum flow cytometry panel for deep immunophenotyping of major cell subsets in human peripheral blood. Cytometry A 97:1044-1051. https://doi.org/10.1002/cyto.a.24213

17. Palit S, Heuser C, De Almeida GP, et al (2019) Meeting the challenges of highdimensional single-cell data analysis in immunology. Front Immunol 10:1515. https://doi.org/10.3389/fimmu.2019.01515

18. Becht E, McInnes L, Healy J, et al (2019) Dimensionality reduction for visualizing single-cell data using UMAP. Nat Biotechnol. https://doi.org/10.1038/nbt.4314

19. Amir E ad D, Lee B, Badoual P, et al (2019) Development of a comprehensive antibody staining database using a standardized analytics pipeline. Front Immunol 10:1315. https://doi.org/10.3389/fimmu.2019.01315

20. Melsen JE, van Ostaijen-ten Dam MM, Lankester AC, et al (2020) A comprehensive workflow for applying single-cell clustering and pseudotime analysis to flow cytometry data. J Immunol 205(3):864-871. https://doi.org/10.4049/jimmunol.1901530 